\title{
Model-based Approach of Controller Design for a FOPTD System and its Real Time Implementation
}

\author{
Anil Kumar Sharma \\ Dept. of Applied Electronics and Instrumentation Engineering, A.I.E.M, Mogra, India
}

\begin{abstract}
For SISO (single-input-single-output) models typically used in the process plants, the Internal Model Control (IMC) is shown to supersede the conventional PID controllers and also it becomes much easier to tune than are controllers in a standard feedback control structure. The conventional PID controllers are used in the process plants to a large extent due to their simple structure and its implementation is also easy. A tuning method based on IMC-PID is proposed for stable FOPTD systems. The IMC-PID controller provides a better trade-off between closed loop performance and robustness to model uncertainties with a single tuning parameter. Performance of the controller is evaluated through simulation study as well as from experimental verification on a level process.
\end{abstract}

Keywords: Feedback control, FOPTD, internal model control (IMC), process model, process reaction curve.

\section{Introduction}

Model-based approach is one type of approach in which once the model of the process is known to us we can approximate the controller. A great deal of work that has provided tuning rules on model-based approach based on frequency and time domain approaches have been reported at different time. In the year 1942, Ziegler and Nichols [1] developed tuning parameters which was published in the paper, "Optimum Settings for Automatic Controllers". These tuning rules were based on process reaction curve method. Tuning rules that have been reported by Cohen and Coon [2], Astrom Hagglund [3] and Tyreus and Luyben [4] were also based on process reaction curve method. Thereafter, Garcia, C.E.; and Morari, M [5] worked on Internal model Control and published a paper, "Internal Model Control I. A Unifying Review and Some Results". Because of the popularity of model-based PID tuning methods, Morari, M; along with Rivera, D.E.; and Skogestad, S.; [6] continued to work upon model-based controller design and published a paper, "Internal Model Control.4. PID Controller Design", in the year 1986. Afterwards, model-based approaches became popular among the researches who were engaged in designing of controllers.

A more comprehensive model-based design method, Internal Model Control (IMC), was developed by Morari and coworkers (Garcia and Morari, 1982; Rivera et al., 1986). Internal Model Control (IMC) method is based on an assumed process model (embedded in the controller) and leads to analytical expressions for the controller settings. The IMC approach has the advantage that it allows model uncertainty and tradeoffs between performance and robustness to be considered in a more systematic fashion [7]. Internal Model control technique is a simple and powerful control structure based on assumed process model and relates the controller settings to the model parameters in a straight forward manner. It explicitly takes into account model uncertainty. It allows the designer to trade-off control system performance against control system robustness to process changes and modeling errors. It provides time-delay compensation. The controller can be used to shape both input tracking and disturbance rejection responses. Perfect tracking is achieved despite model-mismatch, as long as the controller is the perfect inverse of the model [8]. IMCs are much easier to tune than the controllers in a standard feedback control system. Although the IMC design procedure is identical to the open-loop control design procedure, the implementation of IMC results in a feedback system.

In the year 2008, Panda, R.C. [9] reported synthesis of PID tuning rules based on the desired closedloop response. In this paper, Panda simplified an expression for the true controller, which contains a time-delay term, using a power series to approximate its value. This true controller is finally rearranged in a suitable manner such that an ideal PID controller is obtained. Because of very good disturbance rejection and set-point tracking capability, Internal Model Control method become very popular to the control engineers and researchers and work on Internal Model Control is still going on.

\section{Mathematical Approach Of IMC Design}

The IMC design procedure is similar to the design procedure that we developed for open-loop controller design earlier. The assumptions we are making is that the model is perfect i.e., The IMC design procedure consists of the following four steps: 
1. Factor the process model into invertible ("good stuff") and noninvertible ("bad stuff"- time delays and RHP zeros) elements.

$$
\tilde{g}_{p}(s)=\tilde{g}_{p+}(s) \tilde{g}_{p-}(s) .
$$

This factorization is performed so that the resulting controller will be stable.

2. Form the idealized IMC controller. The ideal internal model controller is the inverse of the invertible portion of the process model.

$$
\tilde{q}(s)=\tilde{g}_{p-}^{-1}(s)
$$

3. Add a filter to make the controller proper. A transfer function is proper if the order of the denominator polynomial is at least as high as the numerator polynomial.

$$
q(s)=\tilde{q}(s) f(s)=\tilde{g}_{p-}^{-1}(s) f(s)
$$

If it is most desirable to track step set-point changes, the filter transfer function usually has the form

$$
f(s)=\frac{1}{(\lambda s+1)^{n}}
$$

and $n$ is chosen to make the controller proper (or semi proper). If it is most desirable to track ramp set-point changes (often used for batch reactors or transition control problems), then

$$
f(s)=\frac{n \lambda s+1}{(\lambda s+1)^{n}}
$$

4. Adjust the filter tuning parameter to vary the speed of response of the closed-loop system. If the $\lambda$ is "small", the closed-loop system is "fast", if $\lambda$ is "large", the closed-loop system is more robust (insensitive to model error).

If the process model is perfect, then we can write $y(s)=g_{p}(s) q(s) r(s)$

Now we can easily calculate what the output response to a set-point change will be. Substituting Eq.(2.2) into Eq. (2.5) we get

$$
y(s)=g_{p}(s) q(s) r(s)=g_{p}(s) \tilde{q}(s) f(s) r(s)=g_{p}(s) \tilde{g}_{p^{-}}^{-1}(s) f(s) r(s)
$$

If the model is perfect, then

$$
g_{p}(s)=\tilde{g}_{p}(s)=\tilde{g}_{p+}(s) \tilde{g}_{p-}(s)
$$

and we can substitute Eq. (2.7) into Eq. (2.6) to find

which yields

$$
y(s)=\widetilde{g}_{p+}(s) \tilde{g}_{p-}(s) \tilde{g}_{p-}^{-1}(s) f(s) r(s)
$$

$$
y(s)=\tilde{g}_{p+}(s) f(s) r(s)
$$

Equation (2.9) indicates that the bad stuff must appear in the output response. That is, if the open-loop process has a RHP zero (inverse response), then the closed-loop system must exhibit inverse response. Also, if the process has dead time, then dead time must appear in the closed-loop response.

The most common process model is a first-order plus time-delay (FOPTD) transfer function. The design procedure for such system is shown in the next section. In the following section we show how to estimate parameters for common simple models. By far the most commonly used model, for control system design purposes, is the first-order + time delay (FOPTD) model.

\section{IMC-Based PID Design For A First Order Plus A Time Delay Process}

In the closed loop system a test bench named Feedback Basic Process Rig [38-300] has been used as a process model. This is a single loop system, using water as the process fluid, which allows study of the principles of process control using liquid level and flow rates as the process variables to be controlled. The system consists of a completely self-contained, low pressure flowing water circuit supported on a benchmounted panel.

The level control system can be considered as a FOPTD system. The experimental set-up is shown in Fig. 3.1. The level/flow process control system is a single loop system, using water as the process fluid, which allows study of the principles of process control using liquid level and flow rates as the process variables to be controlled.

\subsection{Model Identification}

For model identification, there are two approaches: Process Reaction Curve method and auto tuning technique. We will perform this by using the Process Reaction curve method. 


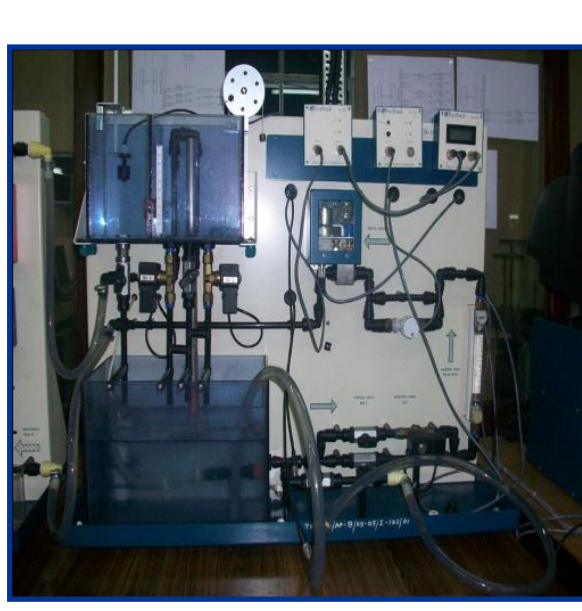

Fig.3.1. Picture of Level/Flow process rig

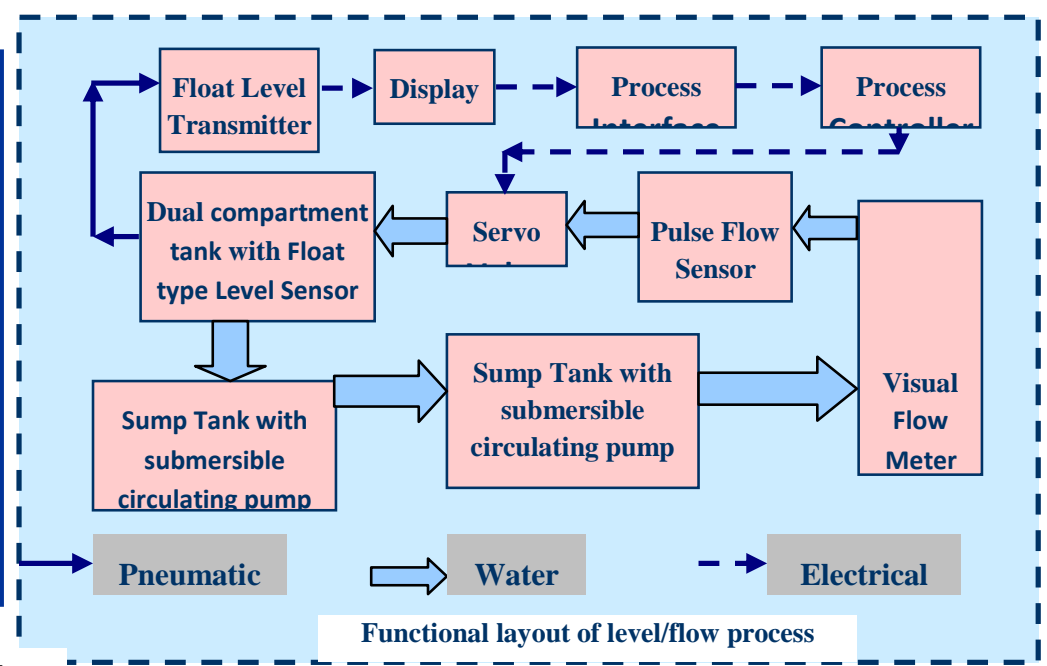

\subsubsection{Application of process reaction curve method on practical process and identification of actual model:}

To get the original process model, we have used the step change method and got a process reaction curve. For getting the process model from this graph we used two-point method. Applying these methods, we have estimated the parameters for first-order + time-delay model and get the original process model. Initially, the process was at a stable condition with a level of height $15 \mathrm{~cm}$ in the upper tank. At this point a step change is applied. Hence, the process reaction curve is obtained and is shown in Fig.3.2.

\subsubsection{Results after applying process reaction curve method:}

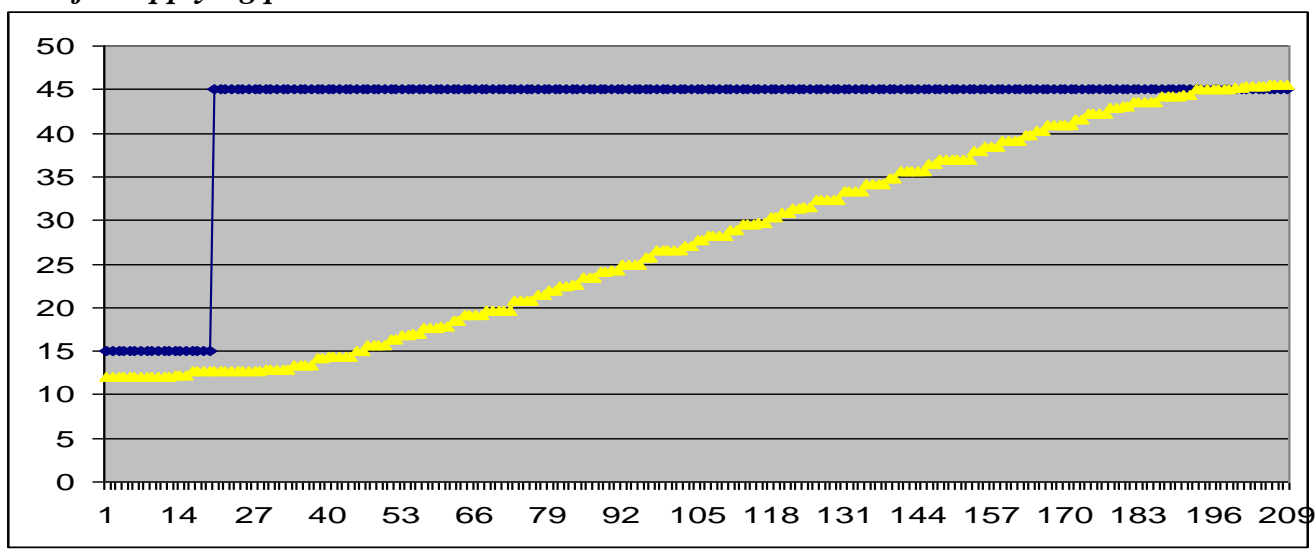

Fig. 3.2 Obtained Process Reaction Curve

$$
k_{p}=\frac{\Delta y}{\Delta u}=\frac{45-15}{100-25}=0.4
$$

Note: Sampling time $=125 \mathrm{msec}$. So the actual process model transfer function is approximated as

$$
g_{p}(s)=\frac{0.4 \cdot e^{-1.75 s}}{4.6 s+1} \text {. }
$$

\section{Experimental Study}

4.1 Different Tuning Rules: Tuning relations given by Rivera et al., Chien, Lee et al., S. Skogestad and R. C. Panda are shown in the following table:

\begin{tabular}{|l|c|c|c|c|}
\hline Paper & $\boldsymbol{K}_{\boldsymbol{c}}$ & $\boldsymbol{\tau}_{\boldsymbol{i}}$ & $\boldsymbol{\tau}_{\boldsymbol{d}}$ & Selection of $\lambda$ \\
\hline $\begin{array}{l}\text { Rivera et } \\
\text { al, } \\
\mathbf{1 9 8 6}\end{array}$ & $\frac{\tau_{p}+0.5 \theta}{K_{p}(\lambda+0.5 \theta)}$ & $\tau_{p}+0.5 \theta$ & $\frac{\tau_{p} \theta}{2 \tau_{p}+\theta}$ & $\lambda>0.8 \theta$ \\
\hline
\end{tabular}


Model-based Approach of Controller Design for a FOPTD System and its Real Time Implementation

\begin{tabular}{|l|c|l|l|l|}
\hline $\begin{array}{l}\text { Chien, } \\
\mathbf{1 9 9 0}\end{array}$ & $\frac{\tau_{p}+0.5 \theta}{K_{p}(\lambda+0.5 \theta)}$ & - & - & $\tau_{p}>\lambda>\theta$ \\
\hline $\begin{array}{l}\text { Lee et al, } \\
\mathbf{1 9 9 8}\end{array}$ & $\frac{\tau_{i}}{K_{p}(\lambda+\theta)}$ & $\tau_{p}+\frac{\theta^{2}}{2(\lambda+\theta)}$ & $\frac{\theta^{2}}{2(\lambda+\theta) \tau_{i}}\left[\tau_{i}-\frac{\theta}{3}\right]$ & $\lambda \geq 0.1 \tau_{p}$ \\
\hline $\begin{array}{l}\text { S. } \\
\text { Skogestad, }\end{array}$ & $\frac{\tau}{K_{p}(\lambda+\theta)}$ & $\min \left[\tau_{p}, 4(\lambda+\theta)\right]$ & - & - \\
\hline $\begin{array}{l}\text { R. C. } \\
\text { Panda, } \\
\mathbf{2 0 0 8}\end{array}$ & $\frac{\tau_{i}}{K_{p}(\lambda+\theta)}$ & $\left(\tau_{p}+\beta\right)+\frac{\theta}{2(\lambda+\theta)}$ & $\frac{\theta^{2}}{2(\lambda+\theta) \tau_{i}}\left[-\frac{\theta}{3}\right]+\frac{\beta \tau_{p}}{\tau_{i}}$ & $\begin{array}{l}\max \left(0.2 \tau_{p},\right. \\
1.7 \theta)\end{array}$ \\
\hline
\end{tabular}

- $\quad \beta$ is a parameter and selection of $\beta$ is based on the following rule: $\beta=\alpha(0.25) \max \left(\tau_{p}, \theta\right)$, where $\alpha$ is a constant $(\alpha=0.1)$.

RESPONSE OF IMC-PID CONTROLLER (RIVERA ET AL., 1986)

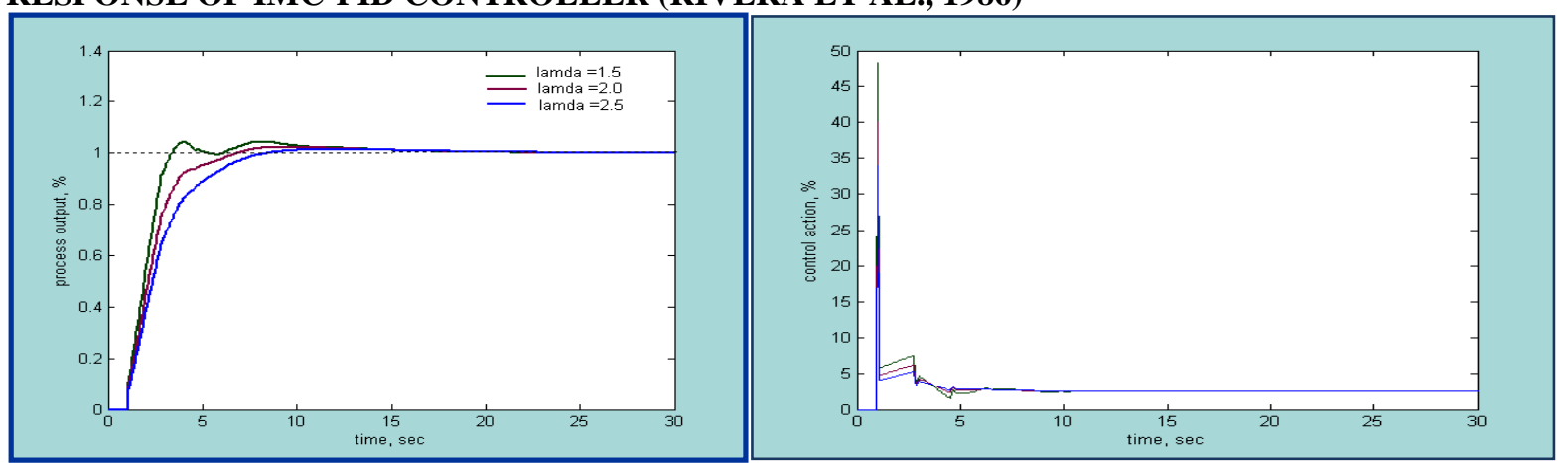

RESPONSE OF IMC-PID CONTROLLER (CHIEN, 1990)

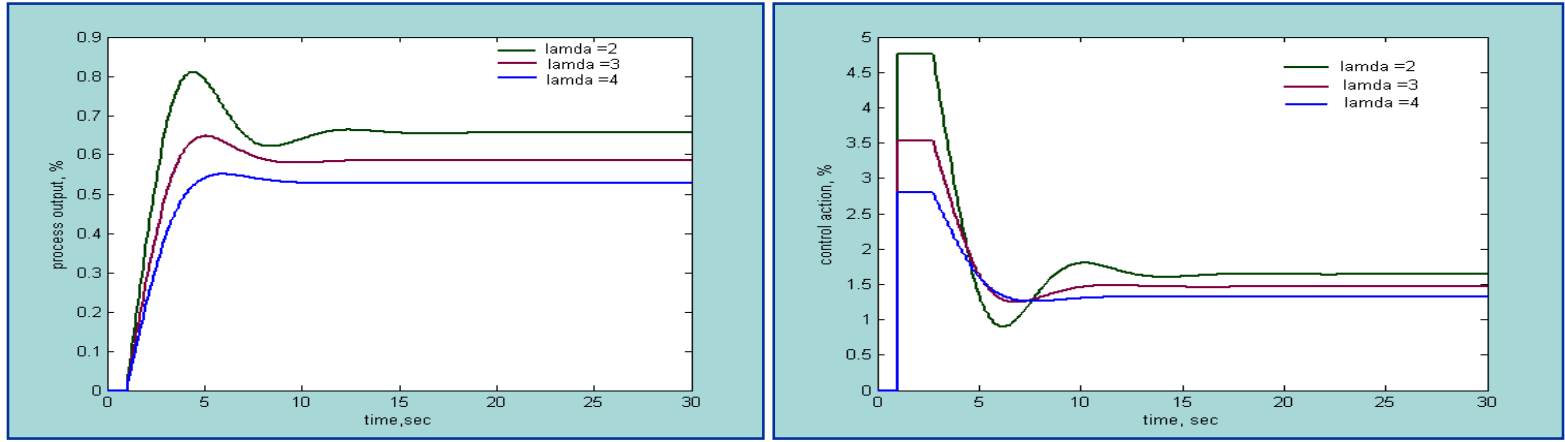

RESPONSE OF IMC-PID CONTROLLER (LEE ET AL., 1998)

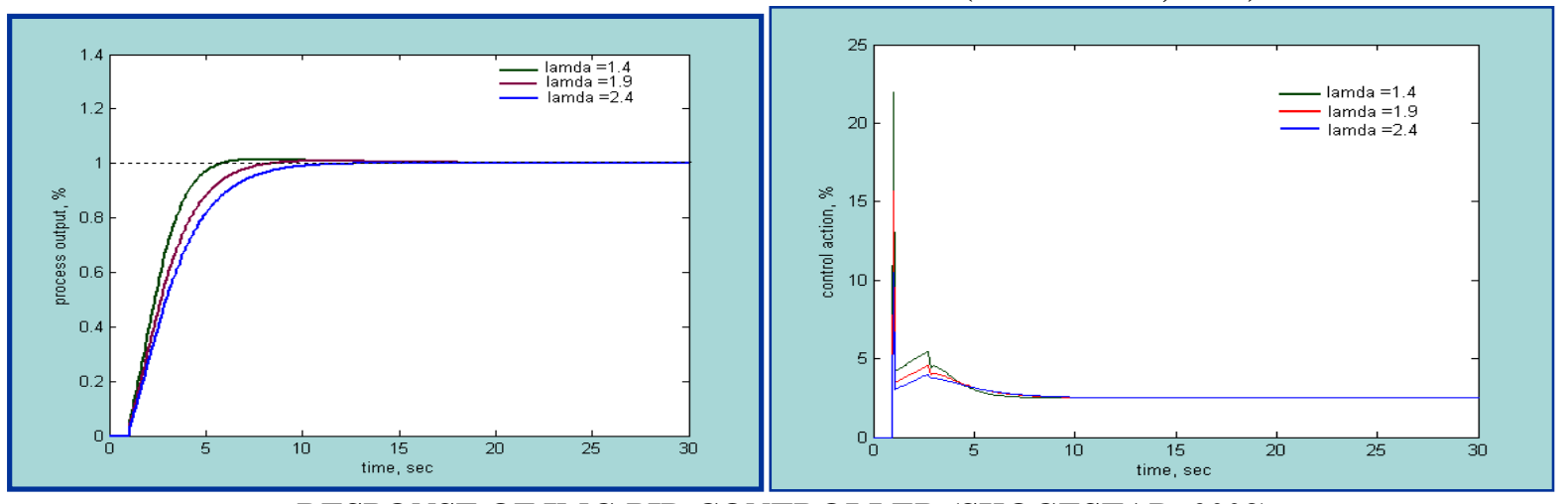

RESPONSE OF IMC-PID CONTROLLER (SKOGESTAD, 2003) 


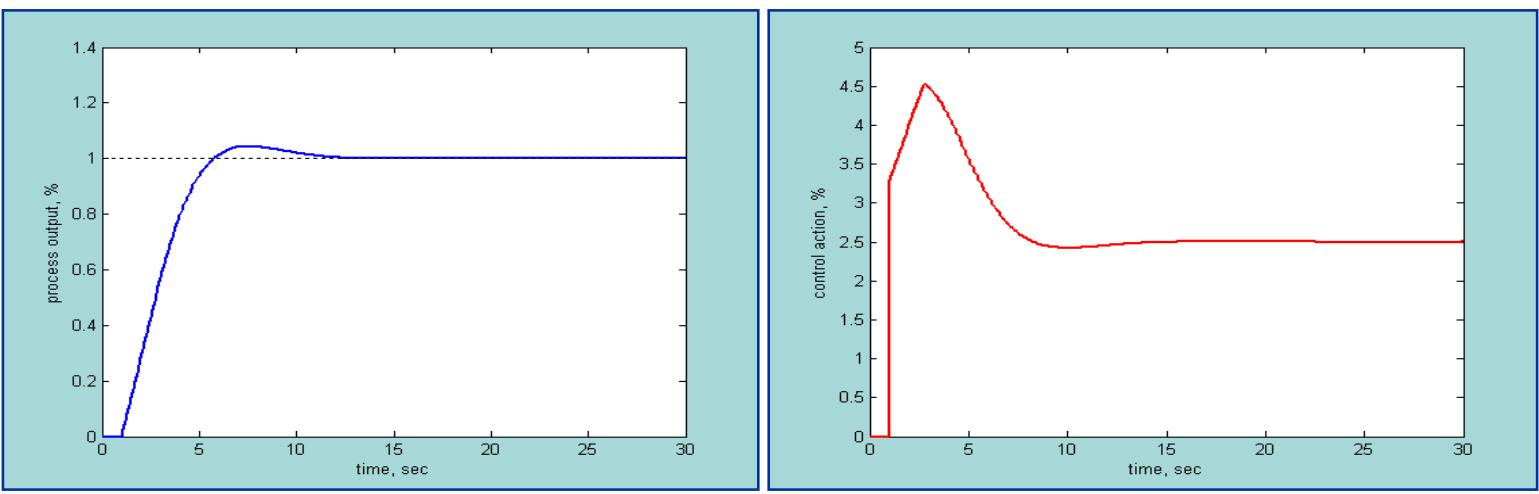

RESPONSE OF IMC-PID CONTROLLER (R. C. PANDA, 2008)
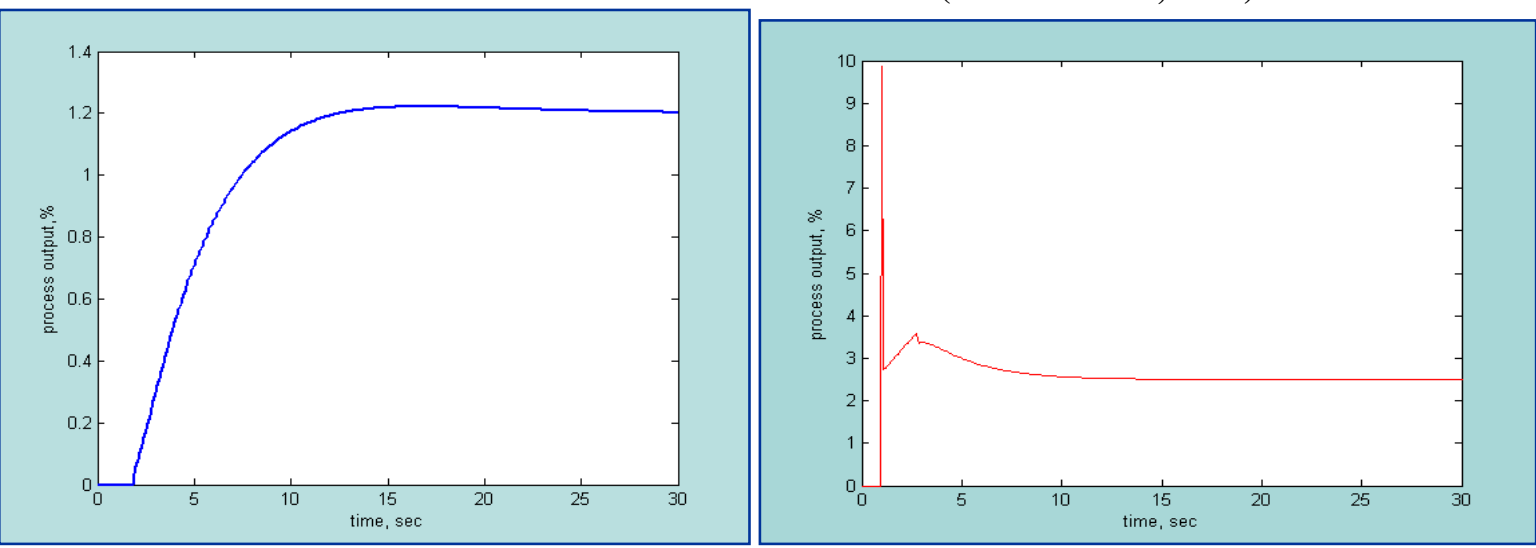

4.2 Comparison Of Different Tuning Rules

\begin{tabular}{|c|c|c|c|c|c|}
\hline Name & $\begin{array}{l}\text { Rise time } \\
\text { (sec) }\end{array}$ & $\begin{array}{l}\text { Settling time } \\
\text { (sec) }\end{array}$ & $\% \mathrm{OS}$ & IAE & ITAE \\
\hline Rivera & 6.81 & 15.38 & 2.45 & 3.00 & 10.35 \\
\hline Chien et al. & 3.69 & 12.26 & 5.79 & 11.42 & 157.2 \\
\hline Lee et al. & 5.84 & 10.33 & 1.23 & 3.81 & 13.06 \\
\hline Skogestad & 5.77 & 12.20 & 4.5 & 3.80 & 12.70 \\
\hline R. C. Panda & 14.41 & 17.73 & 0.29 & 4.71 & 19.61 \\
\hline
\end{tabular}

4.3 Qualitative Performance Of Different Tuning Rules:

\begin{tabular}{|l|l|l|l|l|l|}
\hline Name & $\begin{array}{l}\text { Rise time } \\
(\mathbf{s e c})\end{array}$ & $\begin{array}{l}\text { Settling time } \\
(\mathbf{s e c})\end{array}$ & $\%$ OS & IAE & ITAE \\
\hline Rivera & Medium & Large & Small & Small & Medium \\
\hline Chien et al. & Small & Medium & Medium & Medium & Large \\
\hline Lee et al. & Medium & Medium & Small & Small & Medium \\
\hline Skogestad & Medium & Medium & Medium & Small & Medium \\
\hline R. C. Panda & Medium & Large & Very Small & Small & Medium \\
\hline
\end{tabular}

\section{Conclusion}

This work is based on Level Control System. In our work we have done two parts, i.e., process modeling or model identification and tuning of controllers with Internal Model Control method. For model identification we have used process reaction curve method by which we have got the process model. Finally, for tuning approach, we have used Internal Model Control method and have tried to find out the most acceptable and suitable tuning rules among few standard rules for this Level/Flow Process as well as SIMULINK. We have identified the process model using the process reaction curve method though we can use other methods like relay auto-tuning method and ultimate cycle method. A comparative study is made with recently reported model based tuning schemes. For simplicity first-order + time-delay model has been considered and also such model is a better approximation for many process plants. 


\section{Acknowledgements}

The idea for such a paper arose from discussion with Dr. Chanchal Dey, Asst. Professor, Dept. of Applied Physics, University of Calcutta. He stimulated my interest in controller design. A great thanks to Amit Kumar Sharma, Jr. Engineer, Eastern Railway for his support.

\section{References}

[1] Ziegler, J. G.; Nichols, N. B. Optimum setting for automatic controller. Trans. ASME 1942, 64, 759.

[2] Cohen, G. H.; Coon, G. A. A Theoretical considerations of retarded control. Trans. ASME 1953, 75, 827.

[3] Astrom, K. J.; Hagglund, T. PID Controllers: Theory, Design and Tuning, 2nd Edition; International Society for Measurement and Controls: Research Triangle Park, NC, 1995.

[4] Tyreus, B. D.; Luyben, W. L. Tuning PI Controllers for Integrator/Dead Time Processes. Ind. Eng. Chem. Res. 1992, 32, 2625.

[5] Garcia, C. E.; Morari, M. Internal Model Control I. A Unifying Review and Some New Results, Ind. Eng. Chem. Process Des. Dev., 1982, 21,308.

[6] Rivera, D. E.; Morari, M.; Skogestad, S. Internal Model Control, 4. PID controller design. Ind. Eng. Proc. Des. Dev. 1986, 25, 252.

[7] Seborg, D. E.; Edgar, T. F.; Mellichamp, D. A. Process Dynamics and Control, Second Edition; John Wiley and Sons: New York, 2004.

[8] Bequette. B. W., Process Control Modeling, Design and Simulation, Pearson Education, Inc., New Jersey, 2003.

[9] Panda, R. C. Synthesis of PID Tuning Rule Using the Desired Closed-Loop Response. Ind. Eng. Chem. Res. 2008, 47, 8684-8692.

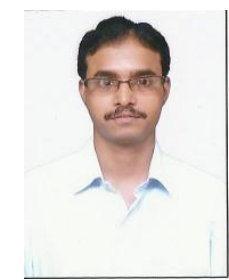

Anil Kumar Sharma is working as an Assistant Professor in the department of Applied Electronics and Instrumentation Engineering, Abacus Institute of Engineering and Management, Mogra, Hooghly. He obtained his M.Tech in Instrumentation and Control Engineering from the department of Applied Physics, University of Calcutta. His area of research includes Intelligent Process Control, Biomedical Instrumentation, Control System and Signal Processing.

E-mail: anil_0181@yahoo.com 